\title{
FINANCING SELECTION METHOD IN DISCIPLINE EVALUATION USING A WEIGHTED INDUCED MODEL
}

\author{
Yufen Chen, Huanhuan Jin, Chao Chen, Chonghui Zhang
}

\section{Introduction}

Managers often have to face kinds of decision-making problems in which a suitable investment alternative has to be evaluated and selected (Zhou et al., 2019; Yan et al., 2017; Rostamzadeh et al., 2017). Such a choice maybe related, for instance, to the technology choose for product development or machine selection for a manufacturing process (Frank et al., 2013). This kind of investment alternatives can be considered a multiple attribute decisionmaking (MADM) problem as it involves a variety of attributes that should be taken into account from finite feasible schemes based on the assessment information provided by decision makers. Due to the uncertain objects and ambiguous human thinking, sometimes it is impossible for decision makers to give all the evaluation values of attributes by exact numbers. The definition of fuzzy set is firstly introduced by Zadeh (1965) to address the uncertainty and ambiguity, which has been widely used in various domains. However, the single membership degree of fuzzy set makes it insufficient to describe complete information of evaluated objects. Later, the intuitionistic fuzzy set (IFS) (Atanassov, 1986), characterized by a membership function and a non-membership function, is introduced to improve the drawback of fuzzy set. So far, the IFS has gained increasing attention and has been widely used in lots of areas, such as economics, engineering technology and management. Recently, Yu and Liao (2016) presented an attractive scientific investigation of the development of IFS from different perspectives. Mehdi et al. (2017) gave a complete literature review on the development of IFS and other type of fuzzy sets in MADM problems from 2001 to 2016.

The previous studies indicated that the IFS is an effective technique to handle complex uncertainty and has shown a rational result in many fields and applications. However, it can only sign exact numbers for the membership and non-membership degrees, but not address the qualitative reference. Inspired by the idea of linguistic terms (Herrera \& Herrera-Viedma, 2000), Wang and Li (2010) first defined the conception of intuitionistic linguistic set (ILS), which integrates the advantages of IFS and linguistic term and is powerful tool to cope with vagueness information. Therefore, more and more researchers have paid their attention to ILS aggregation operators and corresponding MAGDM approaches. For example, Wang and $\mathrm{Li}(2010)$ defined some operational rules on the ILS, including ILS expected values, score function and accuracy function. Liu (2013) studied the application of dependent aggregation operators in ILS situation. Su et al. (2014) investigated ordered weighted distance measures between IL sets and applied them to supplier selection. Xiao and Zhang (2016) introduced the some IL induced distance aggregation operator and investigated its application in financial investment selection. More recently, Liu et al. (2017) presented the weighted induced aggregation operator to facilitate ILS information and utilized it to select low-carbon supplier. Zhang et al. (2017) introduced two Heronian mean IL aggregation operators and applied them in scientific research capacity assessment.

In order to enrich the theory and practice of the ILS, this study continues to study the aggregated method for ILS and its application in investment selection problem. We will develop the IL weighted induced ordered averageweighted averaging (ILWIOWAWA) operator, which integrates the subjective information with the particular attitudinal characters in form of order-induced variables in the same expression to aggregate the ILS information. Some of its major features and special cases are studied. 
Finally, the practicality of the developed operator is tested by a discipline evaluation problem.

The rest of this paper is set out as follows. In next section we give some basic knowledge on the ILS and aggregation operators. Sect. 3 presents the ILWIOWAWA operator and studies its properties. Sect. 4 discusses the applicability and process of the ILWIOWAWA operator in MAGDM problems, and an example concerning disciplines selection is discussed in Sect. 5 . In Sect. 6 we will summarize main results.

\section{Preliminaries}

\subsection{The Intuitionistic Linguistic Set (ILS)}

The linguistic approach is an approximation of technique, which uses linguistic variables to manifest qualitative aspects in term of linguistic values. Let $S=\left\{s_{l} \mid l=0,1, \ldots, t\right\}$ be a linguistic term set with odd cardinality, in which, any linguistic variable, $s_{l}$, has the following characteristic (Xu, 2005):

1. Negation operator: $\operatorname{Neg}\left(s_{i}\right)=s_{t-i}$;

2. Ordered set: $s_{m} \leq s_{n} \Leftrightarrow m \leq n$;

3. Max and min operator: $\max \left(s_{m}, s_{n}\right)=s_{m}$, if $m \geq n ; \min \left(s_{m}, s_{n}\right)=s_{n}$, if $m \leq n$.

Definition 1 (Atanassov, 1986). An IFS $I$ in $Y=\left\{y_{1}, y_{2}, \ldots, y_{n}\right\}$ is given by:

$$
I=\left\{\left\langle y,\left(\mu_{i}(y), v_{i}(y)\right)\right\rangle \mid y \in Y\right\}
$$

where the function $\mu_{I}(y)$ and $v_{I}(y)$ represent the membership degree and non-membership degree of the element $y$ to the set $I$, respectively, $0 \leq \mu_{I}(y)+v_{I}(y) \leq 1$, for all $y \in Y$. $\pi_{I}(y)=\sqrt{1-\left(\mu_{I}(y)\right)^{2}-\left(v_{I}(y)\right)^{2}}$ is defined the degree of indeterminacy of $y$ to the set $I$.

Combining the advantages of the IFS and linguistic term set, Wang and Li (2010) first gave the concept of ILS.

Definition 2. An ILS $A$ in $Y$ is defined as

$$
A=\left\{\left\langle y\left[s_{\theta(y)},\left(\mu_{A}(y), v_{A}(y)\right)\right]\right\rangle \mid y \in Y\right\}
$$

where $\mu_{A}(y)$ and $v_{A}(y)$ is called the membership degree and non-membership degree of the element $y$ to linguistic variable $s_{\theta(y)}$, respectively, $\quad 0 \leq \mu_{A}(y)+v_{A}(y) \leq 1, \quad h_{\theta(y)} \in \bar{S}$. Especially, we call $\pi_{A}(x)=1-\mu_{A}(x)-v_{A}(x)$ as the indeterminacy degree or hesitation degree of $y$ to variable $s_{\theta(y)}$.
From Definitions 1 and 2, it is found that the ILS integrates both the qualitative and quantitative references. This means that ILS enable the decision maker more convenient to address his/her judgment.

Definition 3 (Wang \& Li, 2010). An intuitionistic linguistic number (ILN) is defined as $\tilde{a}=\left\langle s_{\theta(\alpha)},(\mu(\alpha), v(\alpha))\right\rangle$, where $\mu(\alpha)$ and $v(\alpha)$ respectively represents the membership and non-membership degrees of the variable $s_{\theta(y)}$ with $\mu(\alpha), v(\alpha) \in[0,1]$ and $\mu(\alpha)+v(\alpha) \leq 1$.

Let $\tilde{a}_{i}=\left\langle s_{\theta\left(\alpha_{i},\right.},\left(\mu\left(\alpha_{i}\right), v\left(\alpha_{i}\right)\right)\right\rangle(i=1,2)$ be two ILNs and $\lambda \geq 0$, some operational rules for ILNs are defined by (Wang $\& \mathrm{Li}, 2010$ ):

1. $\tilde{a}_{1}+\tilde{a}_{2}=\left\langle s_{\theta\left(\alpha_{1}\right)+\theta\left(\alpha_{2}\right)},\left(1-\left(1-\mu\left(\alpha_{1}\right)\right)\left(1-\mu\left(\alpha_{2}\right)\right)\right.\right.$, $\left.\left.v\left(\alpha_{1}\right) v\left(\alpha_{2}\right)\right)\right\rangle$;

2. $\tilde{a}_{1} \otimes \tilde{a}_{2}=\left\langle s_{\theta\left(\alpha_{1}\right) \times \theta\left(\alpha_{2}\right)},\left(\mu\left(\alpha_{1}\right) \mu\left(\alpha_{2}\right)\right.\right.$, $\left.\left.v\left(\alpha_{1}\right)+v\left(\alpha_{2}\right)-v\left(\alpha_{1}\right) v\left(\alpha_{2}\right)\right)\right\rangle$;

3. $\lambda \tilde{a}_{1}=\left\langle s_{\lambda \theta\left(\alpha_{1}\right)},\left(1-\left(1-\mu\left(\alpha_{1}\right)\right)^{\lambda},\left(v\left(\alpha_{1}\right)\right)^{\lambda}\right)\right\rangle$.

Based on the expected function $E(\tilde{a})=S_{\theta(\alpha) \times\left[\mu(\alpha)+\frac{1}{2}(1-\mu(\alpha)-v(\alpha))\right]}$ and the accuracy function $H(\tilde{a})=\frac{\theta(\alpha)}{l-1} \times(\mu(\alpha)+v(\alpha))$ of ILN $(\tilde{a})=\left\langle s_{\theta(a)},(\mu(\alpha), v(\alpha))\right\rangle$, Liu (2013) gave some rules to compare ILNs.

Definition 4. If $\tilde{a}_{1}=\left\langle s_{\theta\left(\alpha_{i}\right)},\left(\mu\left(\alpha_{1}\right), v\left(\alpha_{i}\right)\right)\right\rangle$ $(i=1,2)$ are any two ILNs, then:

1. If $E\left(\tilde{a}_{1}\right)>E\left(\tilde{a}_{2}\right)$, then, then $\tilde{a}_{1}$ is bigger than $\tilde{a}_{2}$, denoted by $\tilde{a}_{1}>\tilde{a}_{2}$;

2. If $E\left(\tilde{a}_{1}\right)=E\left(\tilde{a}_{2}\right)$, then

$$
\left\{\begin{array}{l}
H\left(\tilde{a}_{1}\right)>H\left(\tilde{a}_{2}\right) \Rightarrow \tilde{a}_{1}>\tilde{a}_{2} \\
H\left(\tilde{a}_{1}\right)=H\left(\tilde{a}_{2}\right) \Rightarrow \tilde{a}_{1}=\tilde{a}_{2}
\end{array}\right.
$$

\subsection{The IOWA and WIOWA Operators}

Definition 5 (Yager \& Filev, 1999). An IOWA is a function $F: R^{n} \times R^{n} \rightarrow R$ that has an associated weighting vector $W=\left(w_{1}, w_{2}, \ldots, w_{n}\right) \in[0,1]^{n}$ with $w_{1}+\ldots+w_{n}=1$ such that:

$$
F\left(\left\langle l_{1}, b_{1}\right\rangle, \ldots,\left\langle l_{n}, b_{n}\right\rangle\right)=\sum_{j=1} w_{j} a_{j}
$$

where $a_{j}$ is $b_{i}$ value induced by the orderinduced variable $l_{i}$.

Due to its superiority of order-induced mechanism, the IOWA operator has been widely used and popularized in recent years. However, the order-induced variables of IOWA are not calculated in aggregation progress, which often cause information loss in the final aggregated results. Recently, Aggarwal (2015) 
introduced an improved IOWA, called the weighted IOWA (WIOWA) operator to conquer this limitation. In the WIOWA operator, the orderinduced variables not only play an inductive role, but also directly involved in the operation of information integration by moderated the associated weights.

Definition 6. A WIOWA is a function: $R^{n} \times R^{n} \rightarrow R$ that has an associated weighting vector $W=\left(w_{1}, w_{2}, \ldots, w_{n}\right) \in[0,1]^{n}$ with $w_{1}+\ldots+w_{n}=1$ such that:

$$
W I O W A\left(\left\langle l_{1}, b_{1}\right\rangle, \ldots,\left\langle l_{n}, b_{n}\right\rangle\right)=\sum_{j=1}^{n} v_{j} a_{j}
$$

where $a_{j}$ is $b_{i}$ value induced by the orderinduced variable $l_{i}$. The weight $v_{j}$ is associated with $w_{j} \in W(j=1,2, \ldots, n)$, as

$$
v_{j}=\frac{w_{j} l_{\sigma(j)}}{\sum_{j=1}^{n} w_{j} l_{\sigma(j)}}
$$

where $(\sigma(1), \ldots, \sigma(n))$ is a permutation relation of $(1, \ldots, n)$ that satisfies the condition $l_{\sigma(j-1)} \geq l_{\sigma(j)}$ for all $j>1$.

\subsection{The ILWIOWA Operator}

The WIOWA is used to infuse real values rather than types of fuzzy numbers. Aggarwal (2015) studied its application in IF environment. Later, Liu et al. (2017) further extended it to the ILS situation and gave a MADM method for supplier selection problems.

Definition 7. For the collection of ILNs $\tilde{a}_{i}=\left\langle s_{\theta_{i}},\left(\mu_{i}, v_{i}\right)\right\rangle(i=1,2, \ldots, n)$, if

$$
\operatorname{ILWIOWA}\left(\left\langle l_{1}, \tilde{a}_{1}\right\rangle, \ldots,\left\langle l_{\mathrm{n}}, \tilde{a}_{\mathrm{n}}\right\rangle\right)=\sum_{j=1}^{n} v_{j} \beta_{j}
$$

where $\beta_{j}$ is $\tilde{a}_{\mathrm{i}}$ value induced by the $l_{i}$, and the weight $v_{j}$ is determined by $w_{j}$ according to Eq. (5), $W=\left(w_{1}, w_{2}, \ldots, w_{n}\right) \in[0,1]^{n}$ such that $w_{1}+\ldots+w_{n}=1$, then the ILWIOWA is called the intuitionistic linguistic WIOWA operator.

Although the WIOWA and the ILWIOWA operators have improved the rule of the orderinduced variables, they fail to take into account the importance of the ILSs' principal component in the aggregation progress, which may lead to loss subjective information in some situations. In order to overtake this limitation, a new aggregation method for ILS will be discussed in next section.

\subsection{ILWIOWA Weighted Average Operator}

The ILWIOWA weighted average (ILWIOWAWA) operator is an integrated method that combines the key advantages of the WIOWA and weighted average to aggregate the IL information. Therefore, it is able to consider both the complex attitude of decision makers and subjective information of the evaluated objects in decision-making problems. It can be defined as follows.

Definition 8. Let $\tilde{a}_{i}=\left\langle s_{\theta_{i}},\left(\mu_{i}, v_{i}\right)\right\rangle$ $(i=1,2, \ldots, n)$ be a group of ILNs to be aggregated, $\omega=\left(\omega_{1}, \omega_{2}, \ldots, \omega_{n}\right)$ is the weight vector of them, which meets $\omega_{i} \in[0,1]$ and $\sum_{i=1}^{n} \omega_{i}=1$. An ILWIOWAWA operator is defined as:

$$
\operatorname{ILWIOWAWA}\left(\left\langle l_{1}, \tilde{a}_{1}\right\rangle, \ldots,\left\langle l_{n}, \tilde{a}_{n}\right\rangle\right)=\sum_{j=1}^{n} \hat{v}_{j} \beta_{j}(7)
$$

where $\beta_{j}$ is $\tilde{a}_{\mathrm{i}}$ value induced by the orderinduced variable $l_{i}$, the combined weight is given as $\hat{v}_{j}=\delta v_{j}+(1-\delta) \omega_{j}$ with $\delta \in[0,1]$ represents the relative importance of the WIOWA method and $\omega_{j}$ the weight (WA) $\omega_{i}$ ordered according to $\beta_{j}$, that is, according to the $j$-th largest value of $l_{i}$, and the weight $v_{j}$ associated with $w_{j} \in W$ $(j=1,2, \ldots n)$, is determined by Eq. (5).

Given the ILWIOWAWA operator is a unification of two aggregation concepts, namely WIOWA and WA, it can be considered a part related to WIOWA operators and the WA operators separately. As a result, we have the following formulation of the ILWIOWAWA operator:

Definition 9. Let $\tilde{a}_{i}=\left\langle s_{\theta_{i}},\left(\mu_{i}, v_{i}\right)\right\rangle$ $(i=1,2, \ldots, n)$ be a group of ILNs to be aggregated, $\omega=\left(\omega_{1}, \omega_{2}, \ldots, \omega_{n}\right)$ is the weight vector of them, which meets $\omega_{i} \in[0,1]$ and $\sum_{i=1}^{n} \omega_{i}$. An ILWIOWAWA operator is defined as:

$$
\begin{aligned}
& \text { ILWIOWAWA }\left(\left\langle l_{1}, \tilde{a}_{1}\right\rangle, \ldots,\left\langle l_{n}, \tilde{a}_{n}\right\rangle\right)= \\
& =\delta \sum_{i=1}^{n} v_{i} \beta_{i}+(1-\delta) \sum_{i=1}^{n} \omega_{i} \tilde{a}_{i}
\end{aligned}
$$

where $\beta_{j}$ is $\tilde{a}_{i}$ value induced by the order-induced variable $l_{i}$, and the weight $v_{j}$ is determined by $w_{j} \in W$, as defined in the Eq. (5), $\lambda \in[0,1]$. Obviously, if $\delta=1$, we get the ILWIOWA operator and if $\delta=0$, the ILWA operator.

Furthermore, based on the approaches mentioned in the recent literature (Merigó 
\& Gil-Lafuente, 2013; Zeng, 2016; Zeng et al., 2016), we can get a series of special cases of IL aggregation operators by signing different weighting schemes (vectors) on the ILWIOWAWA operator, such as the IL induced OWA (ILIOWA), the IL ordered weighted averaging (ILOWA), the window-ILWIOWAWA and the Olympic-ILWIOWAWA operators.

Next, a numerical case is given to show the aggregated process of the ILWIOWAWA operator.

Example 1. Let $\alpha_{1}=\left\langle s_{4},(0.3,0.4)\right\rangle$, $\left.\alpha_{2}=\left\langle s_{6},(0.5,0.4)\right\rangle, \quad \alpha_{3}=\left\langle s_{3},(0.2,0.5)\right\rangle\right) \quad$ and $\alpha_{4}=\left\langle s_{4},(0.6,0.3)\right\rangle$ be a collection of ILNs defined in the linguistic set $S=\left\{s_{1}, s_{2}, s_{3}, s_{4}, s_{5}, s_{6}, s_{7}\right\}$, and their weight vector is $\omega=(0.2,035,0.3,0.15)$. Assume the associated order-induced variables and weighted vector of the ILWIOWAWA operator are $L=(7,3,5,8)$ and $W=(0.1,0.4,0.3,0.2)$, respectively. First we recorder the aggregated argument in the form $\left(l_{i}, \tilde{a}_{l}\right)$ according to the values $l_{i}$ :

$$
\begin{gathered}
\left(\left(8,\left\langle s_{3},(0.2,0.5)\right\rangle\right),\left(7,\left\langle s_{4},(0.3,0.4)\right\rangle\right),\right. \\
\left.\left.\left(5,\left\langle s_{3},(0.2,0.6)\right\rangle\right)\right),\left(3,\left\langle s_{6},(0.5,0.4)\right\rangle\right)\right) .
\end{gathered}
$$

In order to calculate the combined weight $\hat{v}_{j}(j=1,2,3,4)$, we should first calculate the moderated weight $v_{j}(j=1,2,3,4)$ :

$$
v_{1}=\frac{0.2 \times 8}{(0.2 \times 8)+(0.1 \times 7)+(0.3 \times 5)+(0.4 \times 5)}=0.276 \text {. }
$$

Similarly, we can get $v_{2}=0.121, v_{3}=0.258$, $v_{4}=0.345$.

Let $\delta=0.4$, the combined weight $\hat{v}$ $(j=1,2,3,4)$ is obtained by the Definition 6 :

$$
\begin{aligned}
& \hat{v}_{1}=0.4 \times 0.276+0.6 \times 0.2=0.2304, \\
& \hat{v}_{2}=0.4 \times 0.121+0.6 \times 0.35=0.2584, \\
& \hat{v}_{3}=0.4 \times 0.258+0.6 \times 0.3=0.2832, \\
& \hat{v}_{4}=0.4 \times 0.345+0.6 \times 0.15=0.228 .
\end{aligned}
$$

Therefore, we obtain the result aggregated by the ILWIOWAWA operator:

$$
\begin{aligned}
& \text { ILWIOWAWA } \left.\left(\left(8,\left\langle s_{3},(0.2,0.5)\right\rangle\right),\left(7,\left\langle s_{4},(0.3,0.4)\right\rangle\right),\left(5,\left\langle s_{3},(0.2,0.6)\right\rangle\right)\right),\left(3,\left\langle s_{6},(0.5,0.4)\right\rangle\right)\right) \\
& =0.2304 \otimes\left\langle s_{3},(0.2,0.6)\right\rangle \oplus 0.2584 \otimes\left\langle s_{4},(0.3,0.4)\right\rangle \oplus 0.2832 \otimes\left\langle s_{3},(0.2,0.6)\right\rangle \oplus 0.228 \otimes\left\langle s_{6},(0.5,0 .\right. \\
& =\left\langle s_{3.9424},(0.3057,0.4723)\right\rangle
\end{aligned}
$$

The aggregation of ILNs can also be proceeded by Eq. (8). In order to draw a comparison, the aggregation results of intuitionistic linguistic IOWA (ILIOWA) operator is given as follows:

$$
\begin{aligned}
& \left.I L W I O W A W A\left(\left(8,\left\langle s_{3},(0.2,0.5)\right\rangle\right),\left(7,\left\langle s_{4},(0.3,0.4)\right\rangle\right),\left(5,\left\langle s_{3},(0.2,0.6)\right\rangle\right)\right),\left(3,\left\langle s_{6},(0.5,0.4)\right\rangle\right)\right) \\
& =0.4 \otimes\left(0.1 \otimes\left\langle s_{3},(0.2,0.6)\right\rangle \oplus 0.4 \otimes\left\langle s_{4},(0.3,0.4)\right\rangle \oplus 0.3 \otimes\left\langle s_{3},(0.2,0.6)\right\rangle \oplus 0.2 \otimes\left\langle s_{6},(0.5,0.4)\right\rangle\right) \\
& \left.\oplus 0.6 \otimes\left(0.2 \otimes\left\langle s_{4},(0.3,0.4)\right\rangle \oplus 0.35 \otimes\left\langle s_{6},(0.5,0.4)\right\rangle \oplus 0.3 \otimes\left\langle s_{3},(0.2,0.5)\right\rangle\right) \oplus 0.15 \otimes\left\langle s_{4},(0.6,0.3)\right\rangle\right) \\
& ==\left\langle s_{3.9424},(0.3057,0.4723)\right\rangle
\end{aligned}
$$

Obviously, the aggregation results are same for both methods.

It is noticed that if the weighting vector doesn't satisfy with the normalization, i.e., $\hat{V}=\sum_{j=1}^{n} \hat{v}_{j} \neq 1$, then the ILWIOWAWA operator should formed as following:

$$
\begin{aligned}
& \operatorname{ILWIOWAWA}\left(\left\langle l_{1}, \tilde{a}_{1}\right\rangle, \ldots,\left\langle l_{n}, \tilde{a}_{n}\right\rangle\right)= \\
& =\frac{\delta}{V_{s}} \sum_{j=1}^{n} v_{j} \beta_{j}+\frac{(1-\delta)}{W_{s}} \sum_{i=1}^{n} \omega_{i} \tilde{a}_{i}
\end{aligned}
$$

where $V_{s}=\sum_{j=1}^{n} v_{j}$ and $W_{s}=\sum_{i=1}^{n} \omega_{i}$.
According to the ILNs' operational rules described earlier, the above theorem can be driven as follows.

Theorem 1. Let $\tilde{a}_{i}=\left\langle s_{\theta_{i}},\left(\mu_{i}, v_{i}\right)\right\rangle$ $(i=1,2, \ldots, n)$ be a set of ILNs, the aggregation result of the ILWIOWAWA is still an ILN.

The ILWIOWAWA operator features the general desirable properties aggregation operators should have, such as monotonicity, boundedness, idempotency, nonnegativity and reflexivity. These aforementioned properties can be proven by the next theorems: 
Theorem 2. (Monotonicity). Let $\left(\tilde{a}_{1}, \tilde{a}_{2}, \ldots \tilde{a}_{n}\right)$ and $\left(\tilde{a}_{1}^{\prime}, \tilde{a}_{2}^{\prime}, \ldots \tilde{a}_{n}^{\prime}\right)(i=1,2, \ldots, n)$ be two set of ILNs, if $\tilde{a}_{i}^{\prime} \geq \tilde{a}_{i}$ for all $i$, then

$$
\begin{aligned}
& \operatorname{ILWIOWAWA}\left(\left\langle l_{1}, \tilde{a}_{1}^{\prime}{ }_{1}\right\rangle, \ldots,\left\langle l_{n}, \tilde{a}_{n}^{\prime}\right\rangle\right) \geq \\
& \geq \operatorname{ILWIOWAWA}\left(\left\langle l_{1}, \tilde{a}_{1}\right\rangle, \ldots,\left\langle l_{n}, \tilde{a}_{n}\right\rangle\right)
\end{aligned}
$$

Theorem 3. (Idempotency). Ifall $\tilde{a}_{i}=\left\langle s_{\theta_{i}},\left(\mu_{i}, v_{i}\right)\right\rangle$ are equal, i.e., $\tilde{a}_{i}=\tilde{a}=\left\langle s_{\theta_{i}},(\mu, v)\right\rangle(i=1,2, \ldots, n)$ for all $i$, then

$$
\operatorname{ILWIOWAWA}\left(\left\langle l_{1}, \tilde{a}_{1}\right\rangle, \ldots,\left\langle l_{n}, \tilde{a}_{n}\right\rangle\right)=\tilde{a}
$$

Theorem 4. (Bounded). The aggregation result by the ILWIOWAWA operator lies between the max and min aggregated values, i.e.,

$$
\begin{aligned}
& \min \left(\tilde{a}_{i}\right) \leq \operatorname{ILWIOWAWA}\left(\left\langle l_{1}, \tilde{a}_{1}\right\rangle,\right. \\
& \left.\ldots,\left\langle l_{n}, \tilde{a}_{n}\right\rangle\right) \leq \max \left(\tilde{a}_{i}\right)
\end{aligned}
$$

Theorem 5. (Permutation). Let $\left(\left\langle l_{1}, \dot{\tilde{a}}_{1}\right\rangle, \ldots,\left\langle l_{n}, \dot{\tilde{a}}_{n}\right\rangle\right)(i=1,2, \ldots, n) \quad$ is any permutation of $\left(\left\langle l_{1}, \tilde{a}_{1}\right\rangle, \ldots,\left\langle l_{n}, \tilde{a}_{n}\right\rangle\right)$

$$
\begin{aligned}
& \operatorname{ILWIOWAWA}\left(\left\langle l_{1}, \tilde{a}_{1}\right\rangle, \ldots,\left\langle l_{n}, \tilde{a}_{n}\right\rangle\right)= \\
& =\operatorname{ILWIOWAWA}\left(\left\langle l_{1}, \dot{\tilde{a}}_{1}\right\rangle, \ldots,\left\langle l_{n}, \tilde{a}_{n}\right\rangle\right)
\end{aligned}
$$

We can get a more general model of the ILWIOWAWA operator by using the quasiarithmetic means (Merigó, 2011; Zeng, 2017), then we get a more general IL aggregation operator, called the quasi-arithmetic ILWIOWAWA (QILWIOWAWA) operator.

Definition 10. Let $\tilde{a}_{i}=\left\langle s_{\theta_{i}},\left(\mu_{i}, v_{i}\right)\right\rangle$ $(i=1,2, \ldots, n)$ be a group of ILNs to be aggregated, $\omega=\left(\omega_{1}, \omega_{2}, \ldots, \omega_{n}\right)$ is the weight vector of them, which meets $\omega_{i} \in[0,1]$ and $\sum_{i=1}^{n} \omega_{i}=1$. The QILWIOWAWA operator: $\Omega^{n} \times R^{n} \rightarrow \Omega$ that has an associated weighing vector $W$ with $w_{j} \in[0,1]$ and $\sum_{j=1}^{n} w_{j}=1$, such that:

$$
\begin{aligned}
& \text { QILWIOWAWA }\left(\left\langle l_{1}, \tilde{a}_{1}\right\rangle, \ldots,\left\langle l_{n}, \tilde{a}_{n}\right\rangle\right)= \\
& =\delta X^{-1}\left(\sum_{j=1}^{n} v_{j} \beta_{J}\right)+(1-\delta) Y^{-1}\left(\sum_{i=1}^{n} \omega_{i} \tilde{a}_{i}\right)
\end{aligned}
$$

where $\beta_{j}$ is $\tilde{a}_{i}$ value induced by the order-induced variable $l_{i}$, and the weight $v_{j}$ is determined by $w_{j} \in W$, as defined in the Eq. (5), $\lambda \in[0,1]$. An important features of the QILWIOWAWA operator is that it generalizes a number of special cases by employing more complicated formulas in its continuous monotone functions. For example, if $X=a^{\sigma}$ and $Y=a^{9}$, then the QILWIOWAWA reduced to the generalized ILWIOWAWA (GILWIOWAWA) operator.

\subsection{MAGDM with the ILWIOWAWA Operator}

We explore the application of developed operator in MAGDM problem with IL information in this section. Let $A=\left\{A_{1}, A_{2}, \ldots, A_{m}\right\}$ be a set of alternatives to be assessed and $C=\left\{C_{1}, C_{2}, \ldots, C_{n}\right\} \quad$ be the $n$ attributes being considered in the evaluation. Let $\omega=\left(\omega_{1}, \omega_{2}, \ldots, \omega_{n}\right)$ the weights of each attribute $A_{j}$ is $\omega_{j}$, where $0 \leq \omega_{j} \leq 1$ and $\sum_{j=1}^{n} \omega_{j}=1$. Assume $E=\left\{e_{1}, e_{2}, \ldots, e_{k}\right\}$ be the $k$ decision makers with the associated weighting vector $\lambda=\left(\lambda_{1}, \lambda_{2}, \ldots, \lambda_{k}\right) \in[0,1]^{n}$ and the sum of the weights is 1 . Based on the proposed operator, the MAGDM process is given as follows:

Step 1: Identify the IL decision matrix $D^{k}=\left(\tilde{a}_{i j}{ }^{(k)}\right)_{m \times n}$ given by decision maker $e_{t}(t=1,2, \ldots, k)$.

Step 2: Apply the ILWA operator to aggregate the evaluations of the decision makers $E$ based on the weight vector $\lambda$. Then aggregation result forms the IL collective matrix $D=\left(\tilde{a}_{i j}\right)_{m \times n}$, where

$$
\begin{aligned}
& \tilde{a}_{i i}=\operatorname{ILWA}\left(\tilde{a}^{1}{ }_{i i}, \tilde{a}^{2}{ }_{i i}, \ldots, \tilde{a}^{k}{ }_{i i}\right), \\
& (i=1,2, \ldots, m),(j=1,2, \ldots, n) .
\end{aligned}
$$

Step 3: Utilize the Eq. (5) to calculate the weight vector $\hat{v}_{j}=\delta v_{j}+(1-\delta) \omega_{j}$ to be used for the ILWIOWAWA operator.

Step 4: Get the aggregated results for each alternative $A_{i}$ by the ILWIOWAWA operator defined by Eq. 7 or 8 . In this step one can consider some particular cases of the ILWIOWAWA operators that described in Section 3.

Step 5: Rank and select the best one(s) according to the aggregation results obtained in the step 4.

\section{Illustrative Example}

In this section, an example concerning a university that wants to fund some money in the best discipline for developing the level of scientific research. Based on the opinion survey and preliminary screening, four potential disciplines are considered to get the money:

1. $A_{1}$ : Regional Economy;

2. $A_{2}$ : Econometrics; 
3. $A_{3}$ : Industrial Economy;

4. $A_{4}$ : Economic Statistics.

To secure greater benefits and guarantee fairness, the university invite three experts to assess the four alternatives according to the following attributes (criteria):

- $C_{1}$ - Influence of papers;

- $C_{2}$-Amount of projects;
- $C_{3}$ - Number of awards;

- $C_{4}-$ Contribution to society.

Because of the uncertainties associated with the phenomena in analysis, the evaluated information of each alternative with respect to each attribute provided by the experts (whose weight vector is $\lambda=(0.25,0.30,0.45)^{T}$, are expressed in ILNs, given in Tab. 1-3.

\section{Tab. 1: IL decision matrix - Expert 1}

\begin{tabular}{c|c|c|c|c} 
& $C_{1}$ & $C_{2}$ & $C_{3}$ & $C_{4}$ \\
\hline$A_{1}$ & $\left\langle s_{4},(0.2,0.6)\right\rangle$ & $\left\langle s_{3},(0.3,0.4)\right\rangle$ & $\left\langle s_{5},(0.5,0.5)\right\rangle$ & $\left\langle s_{2},(0.2,0.7)\right\rangle$ \\
\hline$A_{2}$ & $\left\langle s_{4},(0.4,0.4)\right\rangle$ & $\left\langle s_{3},(0.5,0.4)\right\rangle$ & $\left\langle s_{3},(0.1,0.7)\right\rangle$ & $\left\langle s_{3},(0.3,0.5)\right\rangle$ \\
\hline$A_{3}$ & $\left\langle s_{3},(0.4,0.6)\right\rangle$ & $\left\langle s_{5},(0.7,0.1)\right\rangle$ & $\left\langle s_{4},(0.5,0.2)\right\rangle$ & $\left\langle s_{1},(0.2,0.8)\right\rangle$ \\
\hline$A_{4}$ & $\left\langle s_{5},(0.5,0.4)\right\rangle$ & $\left\langle s_{3},(0.6,0.3)\right\rangle$ & $\left\langle s_{5},(0.7,0.1)\right\rangle$ & $\left\langle s_{4},(0.3,0.6)\right\rangle$ \\
\hline
\end{tabular}

\section{Tab. 2: IL decision matrix - Expert 2}

\begin{tabular}{c|c|c|c|c} 
& $C_{1}$ & $C_{2}$ & $C_{3}$ & $C_{4}$ \\
\hline$A_{1}$ & $\left\langle s_{4},(0.2,0.7)\right\rangle$ & $\left\langle s_{1},(0.8,0.1)\right\rangle$ & $\left\langle s_{3},(0.7,0.3)\right\rangle$ & $\left\langle s_{6},(0.2,0.8)\right\rangle$ \\
\hline$A_{2}$ & $\left\langle s_{3},(0.3,0.6)\right\rangle$ & $\left\langle s_{4},(0.9,0)\right\rangle$ & $\left\langle s_{3},(0.6,0.3)\right\rangle$ & $\left\langle s_{6},(0.3,0.6)\right\rangle$ \\
\hline$A_{3}$ & $\left\langle s_{2},(0.4,0.6)\right\rangle$ & $\left\langle s_{1},(0.9,0.1)\right\rangle$ & $\left\langle s_{2},(0.8,0.2)\right\rangle$ & $\left\langle s_{1},(0.2,0.7)\right\rangle$ \\
\hline$A_{4}$ & $\left\langle s_{3},(0.5,0.4)\right\rangle$ & $\left\langle s_{2},(0.4,0.3)\right\rangle$ & $\left\langle s_{2},(0.2,0.7)\right\rangle$ & $\left\langle s_{5},(0.7,0.1)\right\rangle$ \\
\hline
\end{tabular}

\section{Tab. 3: IL decision matrix - Expert 3}

\begin{tabular}{c|c|c|c|c} 
& $C_{1}$ & $C_{2}$ & $C_{3}$ & $C_{4}$ \\
\hline$A_{1}$ & $\left\langle s_{2},(0.4,0.6)\right\rangle$ & $\left\langle s_{5},(0.3,0.5)\right\rangle$ & $\left\langle s_{6},(0.5,0.5)\right\rangle$ & $\left\langle s_{2},(0.2,0.8)\right\rangle$ \\
\hline$A_{2}$ & $\left\langle s_{4},(0.8,0.1)\right\rangle$ & $\left\langle s_{6},(0.3,0.6)\right\rangle$ & $\left\langle s_{3},(0.2,0.8)\right\rangle$ & $\left\langle s_{3},(0.1,0.9)\right\rangle$ \\
\hline$A_{3}$ & $\left\langle s_{6},(0.2,0.7)\right\rangle$ & $\left\langle s_{5},(0.4,0.4)\right\rangle$ & $\left\langle s_{1},(0.9,0.1)\right\rangle$ & $\left\langle s_{2},(0.7,0.2)\right\rangle$ \\
\hline$A_{4}$ & $\left\langle s_{6},(0.4,0.3)\right\rangle$ & $\left\langle s_{2},(0.3,0.6)\right\rangle$ & $\left\langle s_{5},(0.4,0.5)\right\rangle$ & $\left\langle s_{2},(0.5,0.5)\right\rangle$ \\
\hline
\end{tabular}




Tab. 4: IL collective decision matrix $D$
\begin{tabular}{c|c|c|c|c} 
& $C_{1}$ & $C_{2}$ & $C_{3}$ & $C_{4}$ \\
\hline$A_{1}$ & $\left\langle s_{3.1},(0.297,0.628)\right\rangle$ & $\left\langle s_{3.6},(0.519,0.292)\right\rangle$ & $\left\langle s_{4.85},(0.571,0.429)\right\rangle$ & $\left\langle s_{3.2},(0.2,0.774)\right\rangle$ \\
\hline$A_{2}$ & $\left\langle s_{3.7},(0.617,0.242)\right\rangle$ & $\left\langle s_{4.65},(0.759,0)\right\rangle$ & $\left\langle s_{3},(0.331,0.577)\right\rangle$ & $\left\langle s_{3.9},(0.216,0.689)\right\rangle$ \\
\hline$A_{3}$ & $\left\langle s_{4.05},(0.317,0.643)\right\rangle$ & $\left\langle s_{3.8},(0.740,0.187)\right\rangle$ & $\left\langle s_{2.05},(0.816,0.146)\right\rangle$ & $\left\langle s_{1.45},(0.486,0.412)\right\rangle$ \\
\hline$A_{4}$ & $\left\langle s_{4.9},(0.457,0.351)\right\rangle$ & $\left\langle s_{2.25},(0.419,0.410)\right\rangle$ & $\left\langle s_{4.1},(0.450,0.370)\right\rangle$ & $\left\langle s_{3.4},(0.533,0.323)\right\rangle$ \\
\hline
\end{tabular}

Source: own

Tab. 5: Order-induced variables
\begin{tabular}{c|c|c|c|c} 
& $C_{1}$ & $C_{2}$ & $C_{3}$ & $C_{4}$ \\
\hline$U$ & 9 & 5 & 8 & 7 \\
\hline
\end{tabular}

Source: own

\begin{tabular}{c|c|c|c} 
Tab. 6: & \multicolumn{3}{c}{ Aggregated results rendered by the ILWIOWAWA operator } \\
& Aggregate result & Score & Rank \\
\hline$A_{1}$ & $\left\langle s_{3.712},(0.321,0.510)\right\rangle$ & $S_{1.507}$ & 4 \\
\hline$A_{2}$ & $\left\langle s_{3.791},(0.498,0)\right\rangle$ & $S_{2.839}$ & 1 \\
\hline$A_{3}$ & $\left\langle s_{2.635},(0.647,0.284)\right\rangle$ & $S_{1.795}$ & 3 \\
\hline$A_{4}$ & $\left\langle s_{3.611},(0.473,0.359)\right\rangle$ & $s_{2.010}$ & 2 \\
\hline
\end{tabular}

First, we utilize the ILWA operator to convert decision information given by the three experts into an IL collective decision matrix $\mathrm{D}=\left(\tilde{a}_{i j}\right)_{m \times n}$, listed in Tab. 4.

In this example, the weight vector of four attributes is assumed to be $\omega=(0.18,0.24,0.32,0.26)^{T}$, and the ILWIOWAWA's weight vector is $W=(0.15,0.20,0.35,0.30)^{T}$ and $\delta=0.4$. In addition, the order-induced variables are given in Tab. 5 .

By employing the information discusses, we can utilize the ILWIOWAWA operator to summarize the rows of the collective matrix and therefore get the overall preference values of the Candidate alternatives. Tab. 6 shows the scores and order ranking with each of alternatives obtained by the ILWIOWAWA operator.

The aggregation results listed in Tab. 6 show that the ranking of the four alternatives is $A_{2}>A_{4}>A_{3}>A_{1}$, and thus the most desirable alternative is $A_{2}$ in this case. It can be seen that the significant features of the ILWIOWAWA operator used to this problem is its ability to incorporate subjective opinions and complex attitude characteristics through 


\begin{tabular}{|c|c|c|c|}
\hline \multirow[t]{2}{*}{ Tab. 7: } & \multicolumn{3}{|c|}{$\begin{array}{l}\text { Scores and ranking of the potential alternatives by the particular cases } \\
\text { of the ILWIOWAWA }\end{array}$} \\
\hline & ILWA & ILIOWA & ILWIOWA \\
\hline$A_{1}$ & $S_{1.599}$ & $S_{1.529}$ & $S_{1.446}$ \\
\hline$A_{2}$ & $S_{2.829}$ & $S_{2.978}$ & $S_{2.845}$ \\
\hline$A_{3}$ & $S_{0.997}$ & $S_{1.814}$ & $S_{1.739}$ \\
\hline$A_{4}$ & $S_{1.998}$ & $S_{1.902}$ & $S_{2.019}$ \\
\hline Ranking & $A_{2}>A_{4}>A_{1}>A_{3}$ & $A_{2}>A_{4}>A_{3}>A_{1}$ & $A_{2}>A_{4}>A_{3}>A_{1}$ \\
\hline
\end{tabular}

Source: own

order-induced variables. Moreover, it can deal with the preferences information that may not be exact but can be expressed in ILNs. On the other hand, it is possible to use some special cases of the ILWIOWAWA operator, such as the ILWA, the ILIOWA and the ILWIOWA operator to derive an overall values of the alternatives. Tab. 7 shows the aggregated results.

Obviously, we get the same best alternative, i.e., $A_{2}$ for all the particular cases. However, the ordering of the all alternatives may be varied according to the particular cases in which they are used. Therefore, this method is very useful and effective as it provides the decision maker(s) more choices to choose different schemes. Thus, they can select the best one(s) according to their beliefs or interests.

\section{Conclusions}

In this paper, an integrated method for aggregating IL information has been developed, namely the ILWIOWAWA operator. The main features of this operator is its ability to handle subjective opinions and complex attitudes that can be represented by order-induced variables. The desired properties of the proposed weighted induced operator has also been studied, which shows the practical and effective application to MAGDM. Moreover, a method and process for tackling MAGDM problems with IL information have been investigated. Finally, a numerical example of financing selection is illustrated to verify the effectiveness and feasibility of the introduced method.

In the future, we are going to study some extensions of the ILWIOWAWA operator by using similarity measures and generalized means. Other application areas, such as management, environment, military and economics, can also be considered in our research work.

This paper is supported by the Projects of National Social Science Fund of China (No. 17CTJ012).

\section{References}

Aggarwal, M. (2015). A new family of induced OWA operators. International Journal of Intelligent Systems, 30(2), 170-205. https://dx.doi.org/10.1002/int.21693.

Atanassov, K. (1986). Intuitionistic fuzzy sets. Fuzzy Sets and Systems, 20(1), 87-96. https://dx.doi.org/10.1016/S01650114(86)80034-3.

Frank, A. G., Souza, D. V. S., Ribeiro, J. L. D., \& Echeveste, M. E. (2013). A framework for decision-making in investment alternatives selection. International Journal of Production Research, 51(19), 5866-5883. https://dx.doi.org/ 10.1080/00207543.2013.802393.

Herrera, F., \& Herrera-Viedma, E. (2000). Linguistic decision analysis: steps for solving decision problems under linguistic information. Fuzzy Sets and Systems, 115(1), 67-82. https://dx.doi.org/10.1016/s0165-0114(99)00024-x.

Liu, J., Wu, X. B., Zeng, S. Z., \& Pan, T. L. (2017). Intuitionistic linguistic multiple attribute decision-making with induced aggregation operator and its application to low carbon supplier selection. International Journal of Environmental Research and Public Health, 14(12), 1451. https://dx.doi.org/10.3390/ijerph14121451. 


\section{Business Administration and Management}

Liu, P. (2013). Some generalized dependent aggregation operators with intuitionistic linguistic numbers and their application to group decision making. Journal of Computer and System Sciences, 79(1), 131-143. https://dx.doi.org/10.1016/j.jcss.2012.07.001.

Mehdi, K. G., Maghsoud, A., Edmundas, K. Z., \& Jurgita, A. (2017). Supplier evaluation and selection in fuzzy environments: a review of MADM approaches. Economic Research-Ekonomska Istraživanja, 30(1), 1073-1118. https://dx.doi.org/10.1080/133167 7X.2017.1314828.

Merigó, J. M. (2011). A unified model between the weighted average and the induced OWA operator. Expert Systems with Applications, 38(9), 11560-11572. https://dx.doi.org/10.1016/j.eswa.2011.03.034.

Merigó, J. M., \& Gil-Lafuente, A. M. (2013). Induced 2-tuple linguistic generalized aggregation operators and their application in decision-making. Information Sciences, 236, 1-16. https://dx.doi.org/10.1016/j. ins.2013.02.039.

Rostamzadeh, R., Esmaeili, A., Nia, A. S., Saparauskas, J., \& Ghorabaee, M. K. (2017). A Fuzzy Aras Method for Supply Chain Management Performance Measurement in SMEs under Uncertainty. Transformations in Business \& Economics, 16(2A), 319-348.

Su, W. H., Li, W., Zeng, S. Z., \& Zhang, C. H. (2014). Atanassov's intuitionistic linguistic ordered weighted averaging distance operator and its application to decision making. Journal of Intelligent \& Fuzzy Systems, 26(3), 1491-1502. https://dx.doi.org/10.3233/IFS-130833.

Wang, J. Q., \& Li, H. B. (2010). Multi-criteria decision-making method based on aggregation operators for intuitionisticlinguisticfuzzy numbers. Control and Decision, 25(10), 1571-1574. https://dx.doi.org/10.3724/SP.J.1087.2010. 02828.

Xu, Z. S. (2005). Deviation measures of linguistic preference relations in group decision making. Omega, 33(3), 249-254. https://dx.doi. org/10.1016/j.omega.2004.04.008.

Yager, R. R., \& Filev, D. P. (1999). Induced ordered weighted averaging operators. IEEE Transactions on Systems Man \& Cybernetics
Part $B, \quad 29(2), \quad 141-150 . \quad$ https://dx.doi. org/10.1109/3477.752789.

Yan, Q., Yin, J., Baležentis, T., Makutėnienè, D., \& Štreimikienè, D. (2017). Energy-related GHG emission in agriculture of the European countries: An application of the Generalized Divisia Index. Journal of Cleaner Production, 164, 686-694. https://dx.doi.org/10.1016/j. jclepro.2017.07.010.

Yu, D. J., \& Li, D. F. (2014). Dual hesitant fuzzy multi-criteria decision making and its application to teaching quality assessment. Journal of Intelligent \& Fuzzy Systems, 27(4), 1679-1688. https://dx.doi.org/10.3233/ IFS-141134.

Yu, D. J., \& Liao, H. C. (2016). Visualization and quantitative research on intuitionistic fuzzy studies. Journal of Intelligent \& Fuzzy Systems, 30(6), 3653-3663. https://dx.doi.org/10.3233/ IFS-162111.

Zadeh, L. A. (1965). Fuzzy sets. Information Control, 8(3), 338-353. https://dx.doi. org/10.1016/S0019-9958(65)90241-X.

Zeng, S. Z. (2016). An extension of OWA operator and its application to uncertain multipleattribute group decision-making. Cybernetics and Systems, 47(5), 363-375. https://dx.doi.org/ 10.1080/01969722.2016.1182362.

Zeng, S. Z. (2017). Pythagorean fuzzy multiattribute group decision making with probabilistic information and OWA approach. International Journal of Intelligent Systems, 32(11), 1136-1150. https://dx.doi.org/10.1002/ int.21886.

Zeng, S. Z., Su, W. H., \& Zhang, C. H. (2016). Intuitionistic fuzzy generalized probabilistic ordered weighted averaging operator and its application to group decision making. Technological and Economic Development of Economy, 22(2), 177-193. https://dx.doi.org/10. 3846/20294913.2014.984253.

Zeng, S. Z., \& Xiao, Y. (2016). TOPSIS method for intuitionistic fuzzy multiple-criteria decision making and its application to investment selection. Kybernetes, 45(2), 282-296. https://dx.doi.org/10.1108/K-04-2015-0093.

Zhang, C. H., Su, W. H., \& Zeng, S. Z. (2017). Intuitionistic Linguistic Multiple Attribute Decision-making Based on Heronian Mean 
Method and Its Application to Evaluation of Scientific Research Capacity. EURASIA Journal of Mathematics Science and Technology Education, 13(12), 8017-8025. https://dx.doi. org/10.12973/ejmste/77933.

Zhou, J., Baležentis, T., \& Streimikiene, D. (2019). Normalized Weighted Bonferroni Harmonic Mean-Based Intuitionistic Fuzzy Operators and Their Application to the Sustainable Selection of Search and Rescue Robots. Symmetry, 11, 218. https://dx.doi. org/10.3390/sym11020218.
Prof. Yufen Chen, Ph.D. Zhejiang Gongshang University College of Statistics and Mathematics

China cyf1688@126.com

Huanhuan Jin, Ph.D. Zhejiang Gongshang University College of Statistics and Mathematics China Jinhh06@163.com

Chao Chen

Zhejiang Gongshang University College of Statistics and Mathematics China hzcc5032@163.com

Chonghui Zhang, Ph.D. Zhejiang Gongshang University College of Statistics and Mathematics China zhangch1988@zjgsu.edu.cn 


\title{
Abstract
}

\section{FINANCING SELECTION METHOD IN DISCIPLINE EVALUATION USING A WEIGHTED INDUCED MODEL}

\author{
Yufen Chen, Huanhuan Jin, Chao Chen, Chonghui Zhang
}

The selection of a suitable financing alternative involves multifarious attributes that should be assessed to provide a basis foundation for decision-making. As an effective generation of intuitionistic fuzzy set and linguistic term, the intuitionistic linguistic set (ILS) has greater power for processing uncertain information during decision-making process. Keep this feature in mind, the main purpose of this paper is to investigate a weighted induced aggregation approach for decisionmaking problem concerning financing selection with complex uncertainty in term of intuitionistic linguistic (IL) information. For this, a new intuitionistic linguistic aggregation operator based on the weighted induced approach, namely the IL weighted induced ordered average-weighted averaging (ILWIOWAWA) operator, is proposed to integrate intuitionistic linguistic information. The special advantage of this operator is that it can integrate the subjective information with the particular attitudinal characters in form of order-induced variables in the same expression during information fusion. Moreover, it is able to deal with uncertain information represented by intuitionistic linguistic set very effectively. Some of its properties and particular cases are mathematically explored. We have proved that it has the properties of monotonicity, boundedness, idempotency, nonnegativity and reflexivity. A further extension of the proposed operator is also represented in term of quasiarithmetic means, then we get the quasi-arithmetic ILWIOWAWA (QILWIOWAWA) operator, which encompasses a very broad class of IL aggregation operators. In addition, based on the developed operator, a procedure for multiple attribute group decision-making (MAGDM) problems has been discussed. Finally, a numerical application related to the discipline evaluation in university is used to show the validity and practicability of the proposed method.

Key Words: Intuitionistic linguistic set, multiple attribute decision-making, induced aggregation operator, financing selection.

JEL Classification: C52, D92, N01.

DOI: 10.15240/tul/001/2019-2-003 